\title{
L'Évacuateur de crues de l'usine hydro-électrique de Rossens avec vannes-secteur doubles
}

\section{The double-sector-gates of the Rossens power dam}

\author{
PAR CUR' F. KOLLBRUNNER \\ Dr. sc. techn., DrRecteyr de la S.A. Conrad Zschokre, \\ Constructions Métalliques, Doetlingen, Suisse
}

English synopsis, p. 418.

\section{Introduction.}

Liusine hydro-electrique de Rossens, au-dessus de Fribourg (Suisse), a été construite pour le compte des Entreprises Electriques Fribourgeoises. L'évacuateur de crues, dont il s'agit dans cet article, doit pouvoir détourner un volume d'eau de $350 \mathrm{~m}^{3} / \mathrm{sec}$, lors d'un niveau de retenue normal à la cote de 677 , en admettant des conditions d'écoulement normales sur le déversoir fortement incliné et à l'entrée du tunnel en courbe. La vanne de réglage à double vantail a été projetée et exécutée par la S. A. Conrad Zschokke, Constructions Métalliques, à Docttingen (Suisse).

\section{Remarques générales.}

D'après les données du maître de l'œuvre, l'ouvrage considéré doit avoir une ouverture libre de 8 mètres et une hauteur de 8 mètres, mesurée de la crête supérieure jusqu'à la retenue désirée. Selon son système breveté, la S. A. Conrad $Z$ schokke a projeté et exécuté une vannesecteur double, semblable à celle de l'usine hydro-électrique de Ruppersvill-Auenstein (1). Les vannes s'appuient sur deux consoles en béton, faisant corps avec les parois latérales du tunnel d'entrée. Les treuils sont disposés sur un petit pont à l'entrée du tunnel et abrités par une cabine appuyée à la paroi d'un rocher voisin. Nous avons ainsi obtenu une construction aux formes simples et esthétiques, s'adaptant fort bien au paysage.

(1) C. F. Kolbbronner. - La vanne-secteur double pour l'usine hydro-électrique de Ruppersvil-Auenstein. Mitteilungen über Forschung und Konstruktion im Stahlbau, Heft No. 3, A. G. Gebrüder Leemann \& Co., Zurich, janvier 1946.

C. F. Kollinunner. - Les vannes de toute dernière construction en Suisse, «La Houille BIanche », No. 1, 1947.

\section{Hydraulique.}

De la suppression des niches pour les vannes résultent des conditions d'écoulement meilleures, sans tourbillons, ni pertes de charge imporlantes. Le bordé convexe et la tòle de déversement ont été choisis en fonction d'un coefficient d'écoulement et qui est bien supérieur à celui des vannes Stoney usuelies.

De plus, ils diminuent, en liaison avec le seuil étanche en bois, le danger de vibration des vannes, dû à l'écoulement supérieur ou inférieur à grande vitesse. Elles assurent donc un fonctionnement rationnel. La tôle de déversement et la vanne inférieure ont ćté prérues de façon à ce qu'aucune partie de la vanne ne soit atteinte par le jel du déversement.

Le chemin maximum de la vanne supérieure est de 3 mètres par construction. La forme de la tòle de déversement a été choisie d'après Creager-Escande (2), afin d'éviter les vibrations dues aux dépressions et afin que la charge soit réduite à son minimum. La pression à différentes cotes a été déterminée par la méthode de Prasil. Les tôles de protection latérales ont été conçues afin de n'être pas débordées au cour's d'un abaissement de 3 mètres de l'élément supérieur. Ces boucliers étan: quelque peu éloignés des piliers, il importait de donner à leur crête frontale une forme favorable au point de vue hydraulique. Nous avons obtenu ainsi un accès aisé de l'air à la lame déversante, ce qui simplifie la construction des piliers et des appuis.

(2) C. F. Kondbrunven et W. Wyss : Anuendung der Potentialtheorie im Stahlwasserbau. Berechnung des Veberfallbleches von Hakenschützen. Mitteilungen über Forschung und Konstruktion im Stahlbau, Heft $\mathrm{Nr}, 6$, A. G. Gebrüder Leemann \& Co., Zurich, juin 1947. 


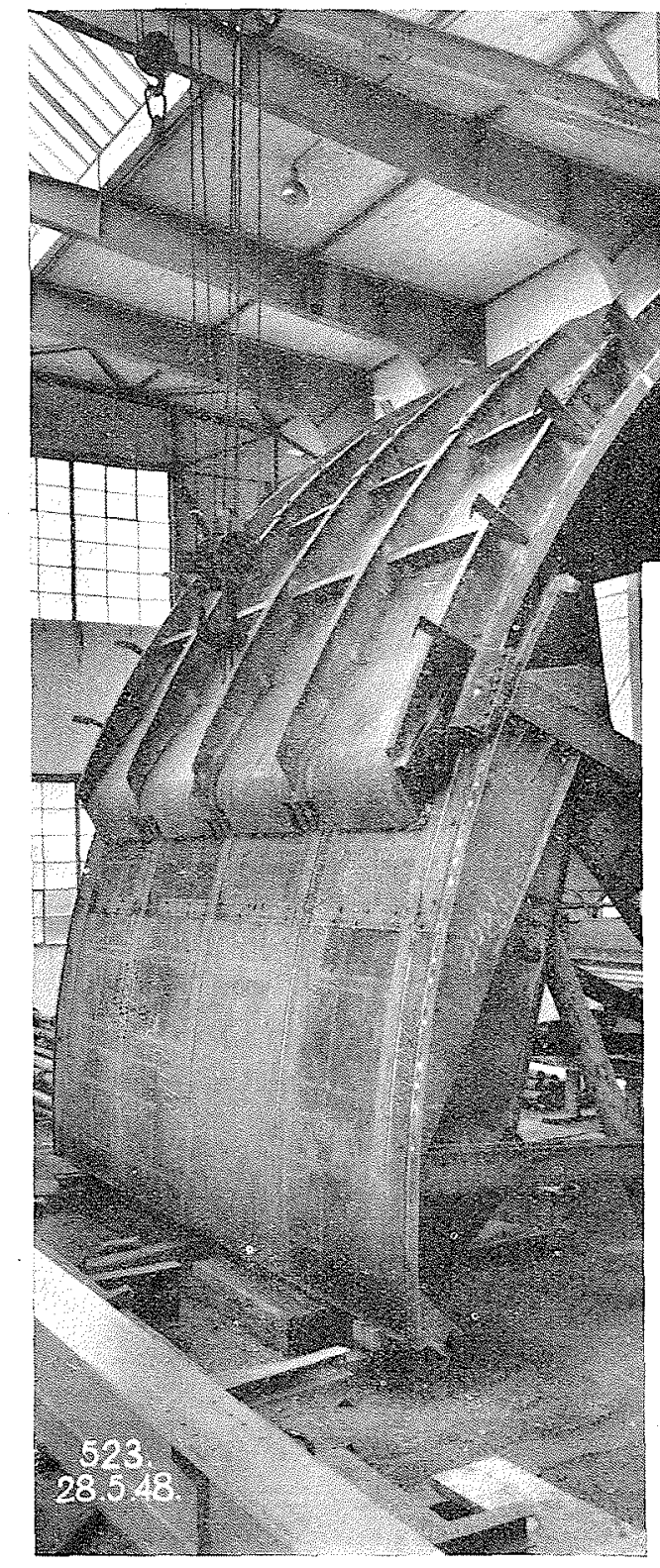

Fig. 2. - Vannes a l'atelier. Vanne supérieure élevée.

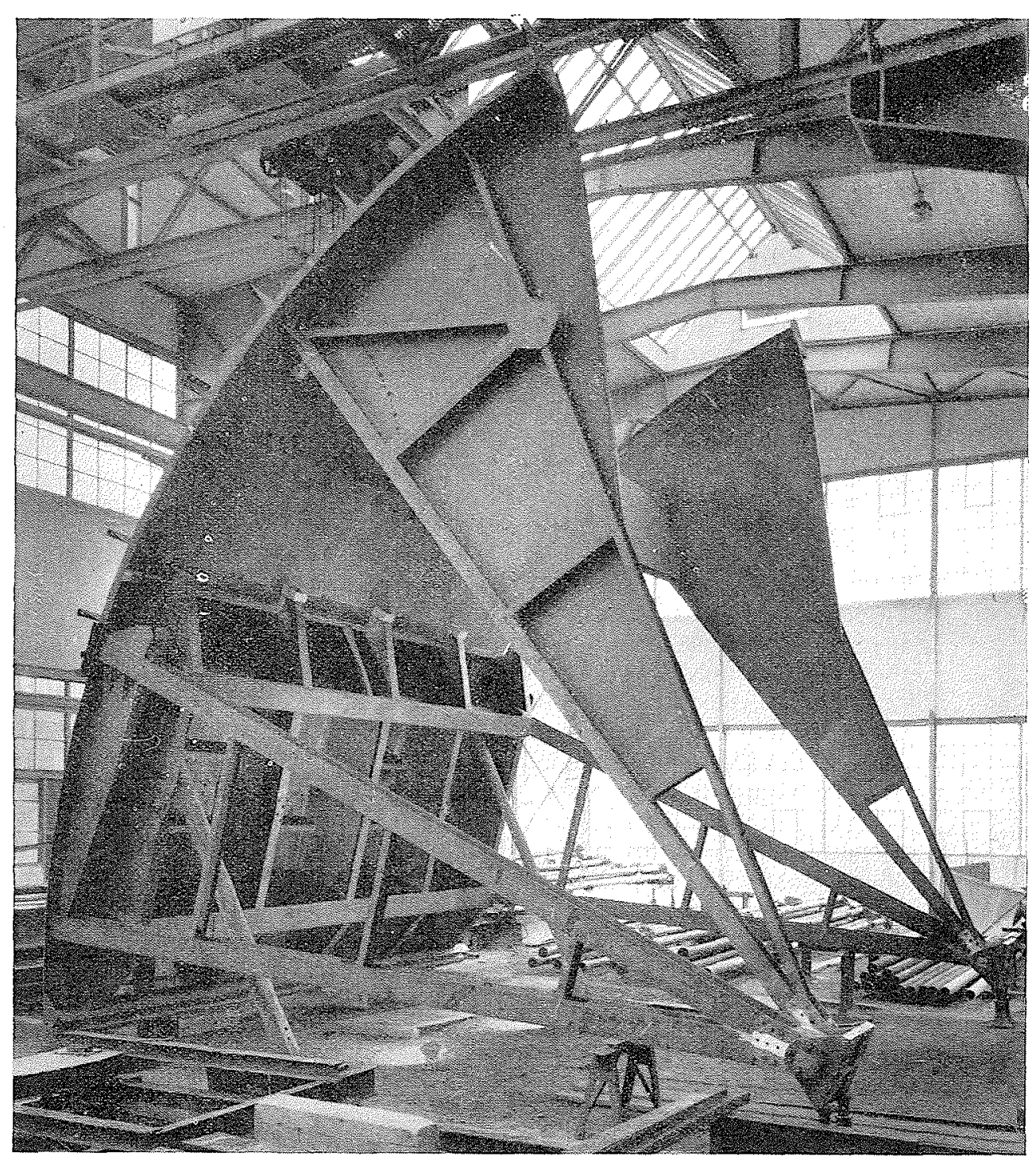

Fig, 1. - Vannes a ridtelien.

Vanne supérieure partiellement élevée. 
Pour les vannes, les piliers ne comportent aucune niche, ni protubérance et offrent des surfaces latérales planes, ce qui est très favorable, tant au point de vue hydraulique qu'au point de vue technique. Ainsi le courant n'est pas dévié el ne fait pas de dépôts. Les parois permettent en outre une fixation aisée des pièces fixes des étanchéités latérales.

Les conditions d'écoulement, lors de l'ouver-
Elles ont été soudées en atelier en des parties aussi grandes que possible, et ont été rivées au montage.

\section{Statique.}

Les constructions métalliques ont été calcuJées d'après les prescriptions sur le calcul, l'execution et l'entretien des constructions métalli-

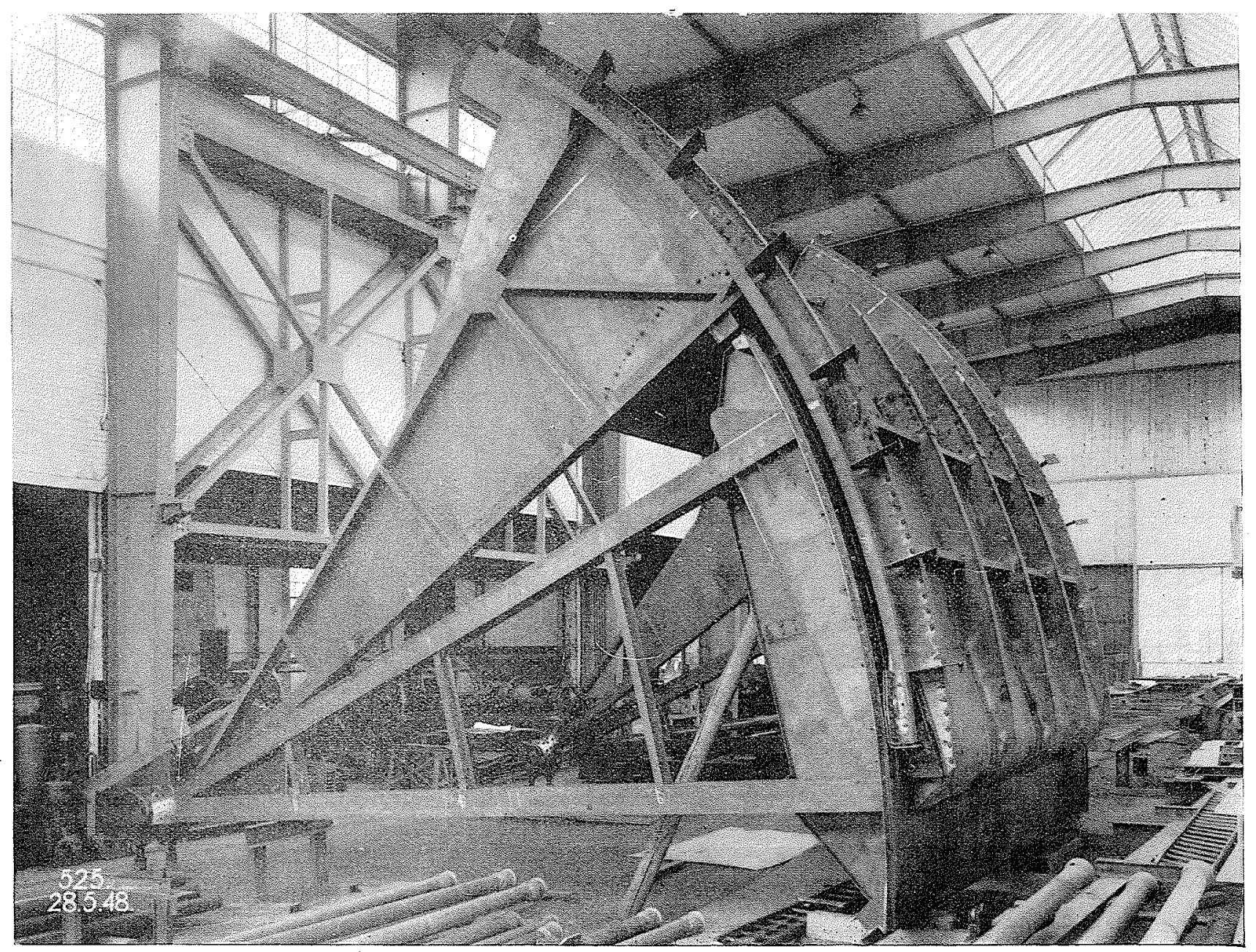

Fig. 3. - Vannes a lótelier. -. Vanne supéricure baissée.

ture de la vanne inférieure, sont favorisées aussi par les profils variables du seuil d'étanchéité et par la construction bombée de la vanne. D'après les expériences du laboratoire de recherches hydrauliques et géotechniques de l'ETH à Zurich, le seuil d'étanchéité a été fixé à $1,4 \mathrm{~m}$ en aval de la crête supérieure du déversoir.

\section{Matériel.}

L'acier de haute qualité n'offre aucun avantage pour une petite portée, et c'est pourquor toutes les vannes ont été construites en acier 37. ques, en béton et bois (S.I.A., Norme 112 de 1935). Les vannes furent calculées pour une pression d'eau de $8,8 \mathrm{~m}$, inclus une marge de $10 \%$, à la retenue maximum, tenant compte des vibrations éventuelles. Le calcul du bordé resulte de la théorie des voiles.

\section{Construction.}

Les dimensions principales des vannes en coupe (centre de rotation, rayon de la vanne, point d'appui) sont déterminées, d'une part, par les considérations les plus favorables au point 


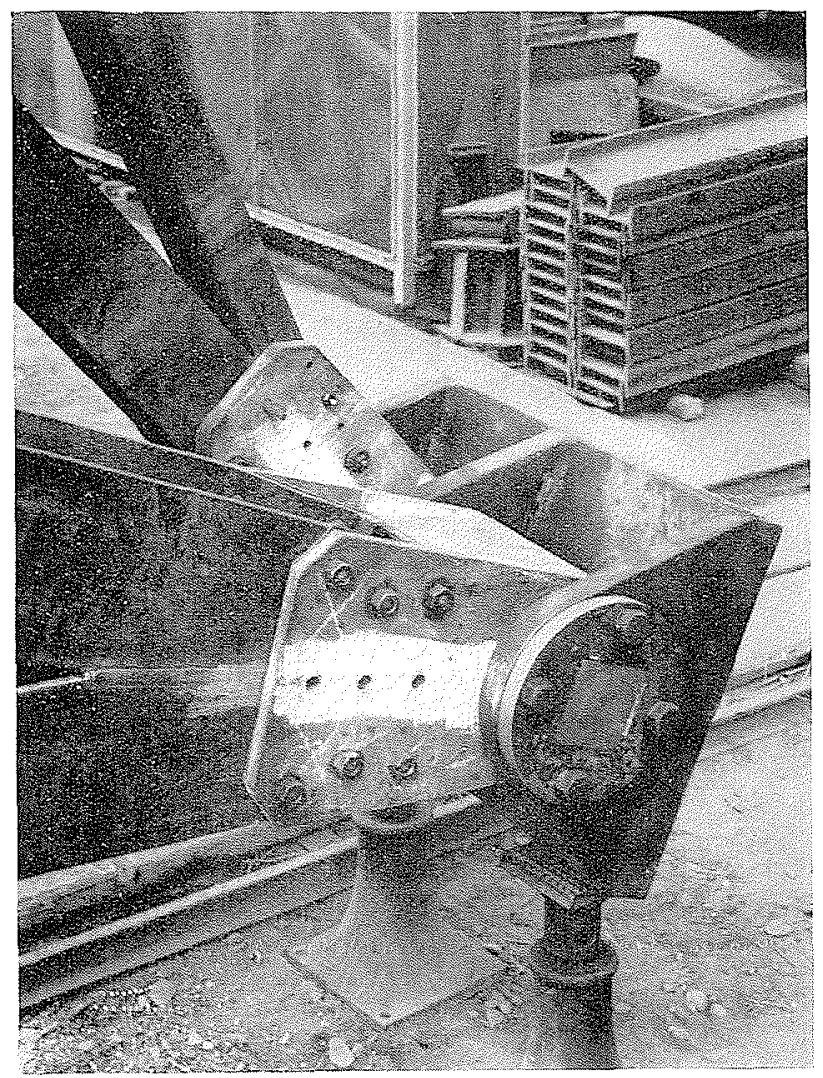

Fig. 4. - Vannes a L'atelier.

Détails des jambages d'appui et de l'appui.

de vue statique et hydraulique, d'autre part par la nécessité de disposer les appuis dans une position inaccessible à l'eau.

Les piliers, aussi étroits que possible, ont été conditionnés par la disposition des treuils, qui ne furent pas montés sur les piliers et appuis, mais sur un petit pont au-dessus du tunnel d'entrée. Cette disposition présente, en outre, les avantages suivants: la protection de l'ensemble des treuils ne nécessite qu'une seule cabine; d'autre part, la position des engrenages et les chaînes de levage, toujours au sec, sont facilement accessibles.

La vanne-secteur brevetée est une vanne double, qui se compose d'une vanne inférieure normale à secteur et d'une vanne supérieure à secteur ayant une section en forme de potence. Les deux vannes se meuvent autour d'un centre de rotation commun. La vanne inférieure a un bordé fortement raidi, s'appuyant aux deux poutres-maîtresses à âme pleine, par l'intermédiaire d'entretoises verticales. Les poutres-maîtresses et les jambages d'appui constituent des cadres à deux articulations. Linexistence théorique de forces latérales permet la suppression des guidages latéraux. Le bordé de la vanne supérieure est raidi verticalement et horizontalement à sa partie inférieure par son extrémité recour- bée et à sa partie supérieure par la tòle de diversement.

La forme à donner à cette tôle, pour ètre avantageuse du point de vue hydraulique, doit être choisie de manière à ce que, lor's du déversement, ia nappe d'eau n'atteigne aucune partie de la vamne, que la charge soil réduite à son minimum et que les sous-pressions soient éliminées. Elant donné la rolation de la vamne, la forme du déversoir déterminée selon Escand: donne pour chaque position une surcharge d'eau minimum, de sorte que les forces à mettre en nuve pour monter la vanne soient sensiblement réduites. Latéralement, la nappe déversante est dirigéc par les tôles de protection.

Toute la charpente métallique de la vanue inférieure, telles que poutrelles, couples et entretoises, est disposée sur la face aval. Les couples et les entretoises de la vanne supérieure, en revanche, sont placés vers l'amont, c'est pourquoi les deux vannes peuvent coulisser l'une devant l'autre.

Le point de suspension de la vanne inférieure se trouve au jambage d'appui a la proximité du bordé. Celui de la vanne supérieure, par construction, est disposć à la partie supérieure des boucliers latéraux.

La vanne inférieure a été concue de telle facon qu'ils ne peul pas se produire de déformations notables, mème si un objet se coince entre

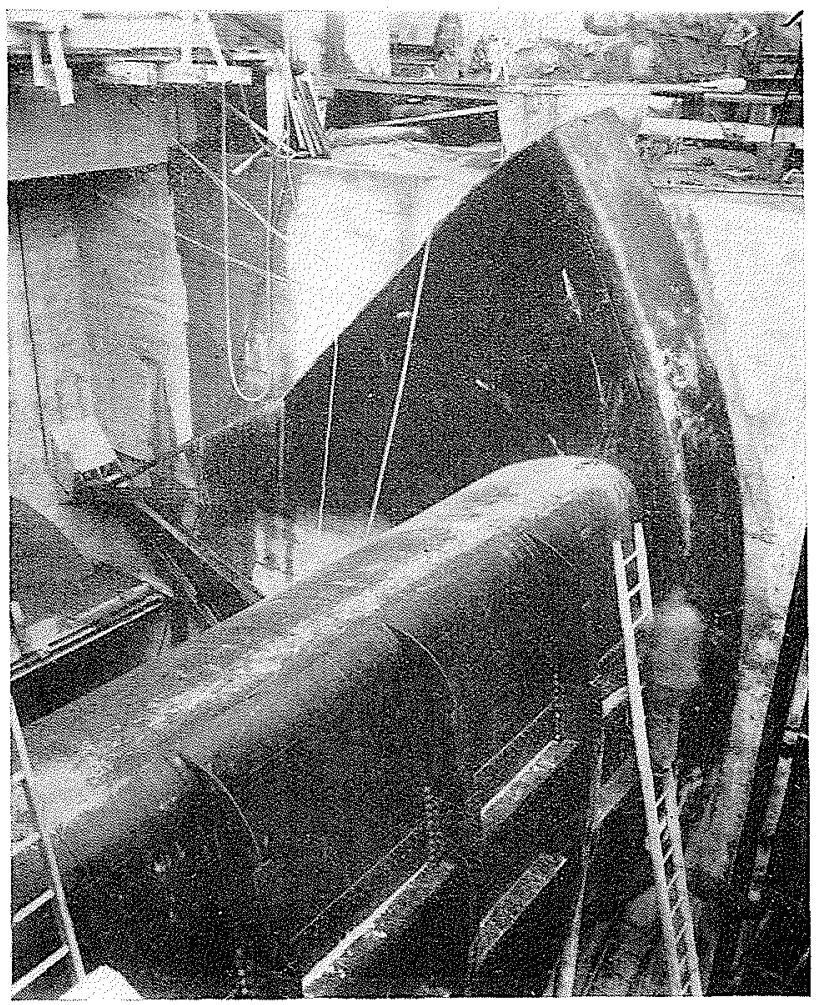

Fig. 5. - Montage des vannes.

Tôle latérale avec jambages d'appui et appuis. 


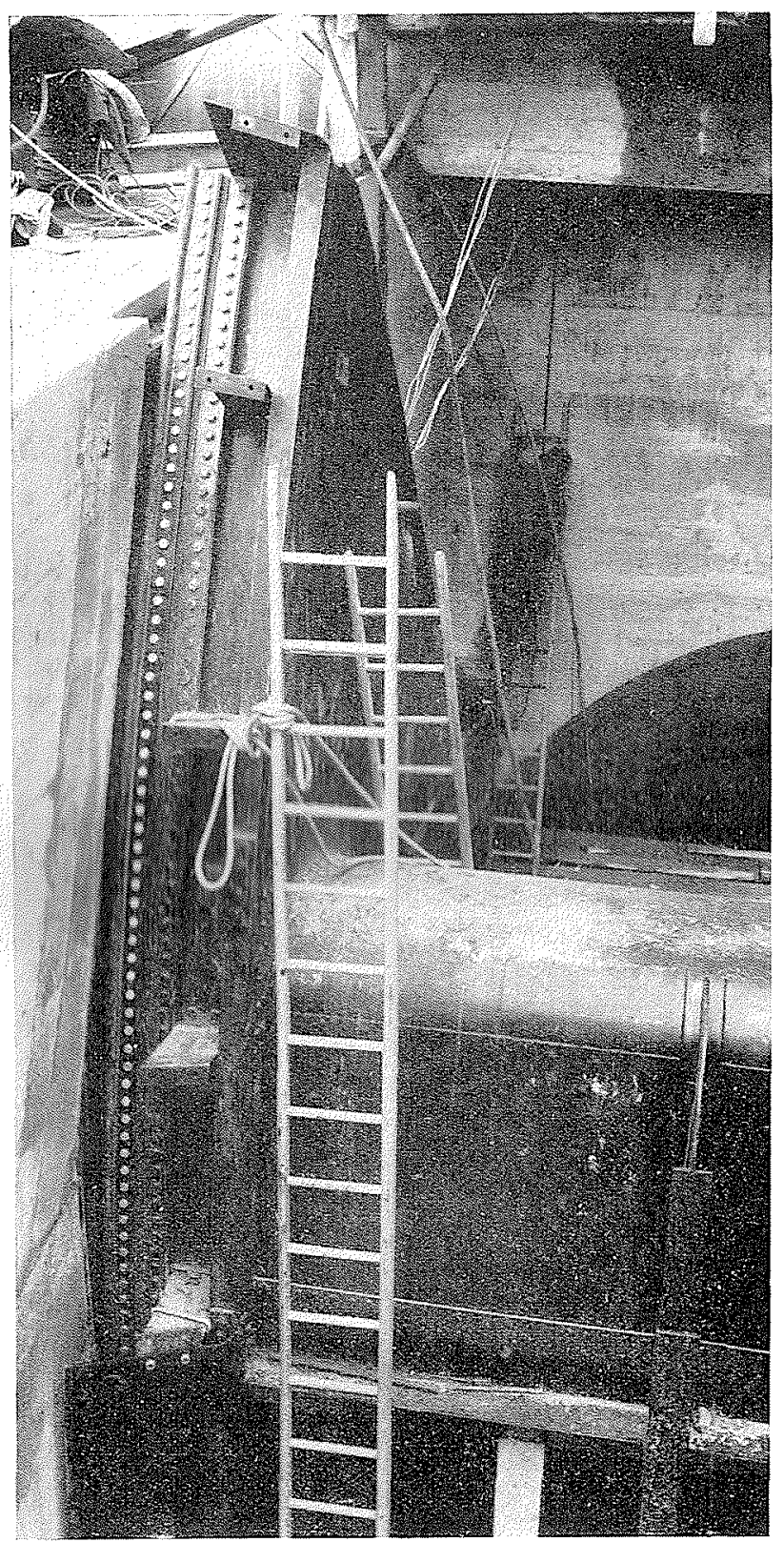

Fre, 6, - Mostage bes varies

A gauche, joints verticaux en caoutchouc.

la vanne et le seuil. La vanne supéricure s'appuie d'une part sur la vanne inférieure, par l'extrémité des poutres verticales, au moyen de cing galets, dautre part sur les consoles, par lintermédiaire des jambages d'appui, raidis par les tôles latérales. La tôle de déversement et la poutre-maitresse forment un raidissement à la torsion encastrée dans les deux boucliers. Celuici a été conçu de facon à prendre les charges verticales dues au poids propre et aux charges hydrauliques, sans nécessiter les crochets de retenue usuels aux vamnes à crochet.

Les appuis se composent d'acier moulé nor- mal et de paliers en bronze. Le graissage provient de la cabine des treuils.

Les avantages au point de vue constructif de la vanne-secteur double, par rapport à la vanne normale, sont dus au gros raidissement de toute la construction, provenant du bordé bombé, ei a la simplicilé des appuis. (Une paire d'appuis robustes, facilement accessibles, remplace les galets de roulement nombreux et difficilement accessibles.) Les étanchéités intermédiaires et latérales sont composées de profils en caoutchouc.

Lors du projet de la vanne, il a été prévu un chauffage des parties mobiles. Dans ce but. deux conduites ont été aménagées dans le revêtement des piliers et une à proximité de l'étanchéité intermédiaire. Celles-ci comprennent des éléments de chauffage électrique.

Toute la construction, composée de profils simples et facilement obtenables, permet un en. tretien facile et bon marché.

Le poids total de la construction métallique est de $35.600 \mathrm{~kg}$ y compris les deux vannes, pieces fixes pour vannes et appuis en acier moulé pour niches et batardeaux).

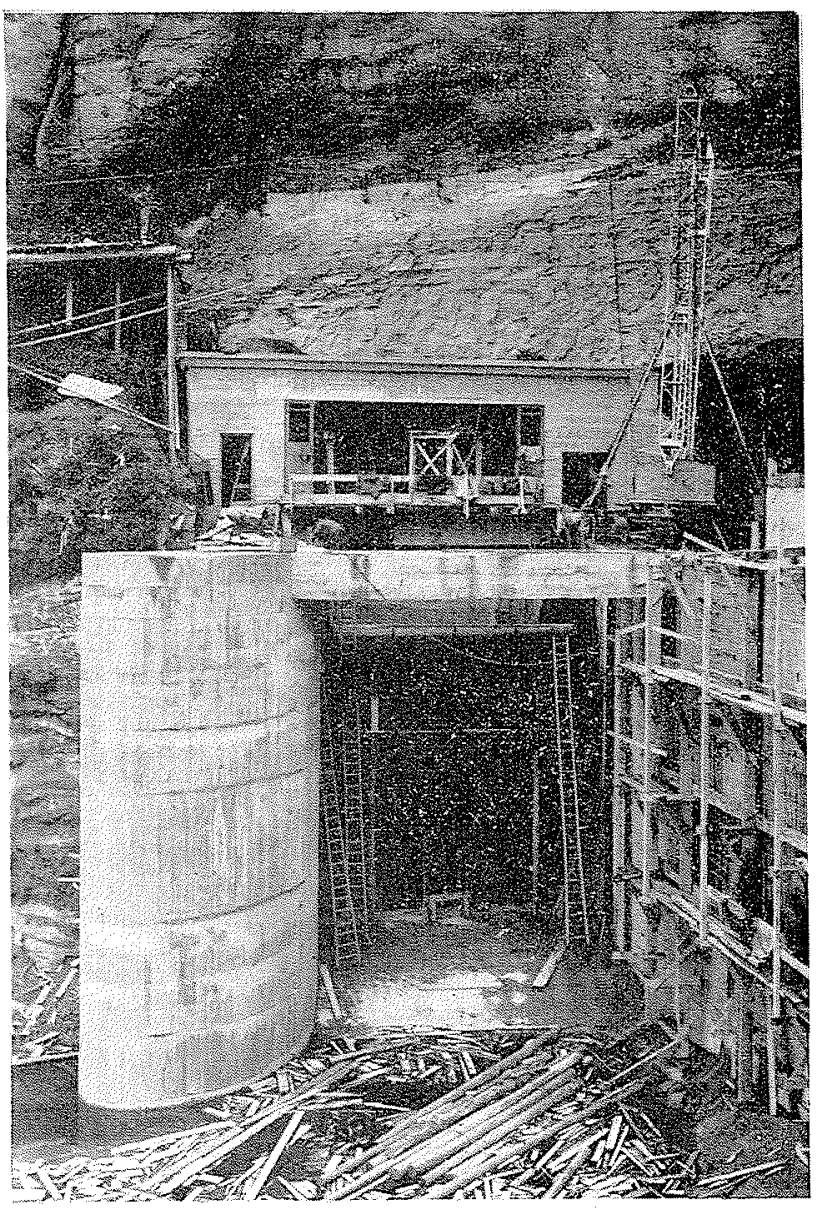

Kig. 7. - VUE DE L'ENTREE DU TUNNEL, avec piliers et cabine des treuils. 